\title{
Rescate del conocimiento etnobotánico para el tratamiento de enfermedades del ganado en el distrito de Leymebamba, Región Amazonas
}

\section{Rescue of ethnobotanical knowledge for the treatment of cattle diseases in the district of Leymebamba, Amazonas Region}

\author{
Fely Enmeline Oclocho García ${ }^{1 *}$, Jesús Rascón ${ }^{1}$, Fernando Corroto $^{1}$
}

\section{RESUMEN}

La ganadería en nuestro país es una actividad que se desarrolla en casi todas las regiones. Su importancia radica en que brinda trabajo y protección a muchas familias campesinas, contribuyendo de esta manera con la bolsa familiar. Sin embargo, en esta actividad encontramos algunas limitaciones que podrían en riesgo la productividad, como es el caso de la salud animal (las diferentes enfermedades que sufre el ganado). Por otro lado se sabe que el Perú desde la antigüedad posee el beneficio de contar con plantas medicinales para todo tipo de dolencias, es por ello que se decidió rescatar los conocimientos etnobotánicos para el tratamiento de enfermedades en la ganadería, y para obtener esta información se aplicaron encuestas semiestructuradas siguiendo el protocolo para la obtención de información etnobotánica y variables socioeconómicas en el distrito de Leymebamba. Los resultados obtenidos nos muestran que las mujeres son las que más uso hacen de las plantas medicinales, así como también la población que se encuentra en el rango de edad de 21 a 40 años. De esta manera se colectaron e identificaron 26 especies de uso medicinal en el ganado, priorizando las hojas como parte usada de estas plantas, la forma de preparación hervida, el modo de administración oral, el lugar de colecta en las chacras en estado silvestre y el uso actual y presente de este conocimiento. Como conclusión negativa se expone que el conocimiento que se tiene sobre el uso e importancia de las plantas medicinales no se está transmitiendo a nivel de rangos de edad dentro de la comunidad.

Palabras claves: Conocimiento etnobotánico, rescate, enfermedades de ganado, plantas medicinales.

\begin{abstract}
Livestock in our country is an activity that takes place in almost all regions. Its importance lies in that it provides work for many peasant families, thus contributing to the family exchange. However, in this activity we found some limitations that could threaten productivity, as is the case of animal health (the different diseases suffered by livestock). On the other hand it is known that Peru since antiquity has the benefit of having medicinal plants for all kinds of ailments, that is why it was decided to rescue ethnobotanical knowledge for the treatment of diseases in livestock, and to obtain this information we used the applied semi-structured surveys following the protocol for obtaining ethnobotanical information and socioeconomic variables in the district of Leymebamba. The results obtained show us that women are the ones that make the most use of medicinal plants, as well as the population that is in the age range of 21 to 40 years. In this way, 26 species of medicinal plant use were collected and identified in the cattle, prioritizing the leaves as a part of these plants, the boiled preparation form, the oral administration mode, the collection place in the farms in the wild and the current and present use of this knowledge. As a negative conclusion, it is stated that the knowledge that we have about the use and importance of medicinal plants is not being transmitted at the level of age ranges within the community.
\end{abstract}

Keywords: Ethnobotanical knowledge, rescue, livestock diseases, medicinal plants.

\footnotetext{
${ }^{1}$ Universidad Nacional Toribio Rodríguez de Mendoza de Amazonas (UNTRM-A), Instituto de Investigación para el Desarrollo Sustentable de Ceja de Selva, Calle Higos Urco N³42-350-356, Calle Universitaria N³04, Chachapoyas, Perú

"Autor de correspondencia.E-mail: foclocho@indes-ces.edu.pe
} 


\section{INTRODUCCIÓN}

La ganadería es una de las actividades socioeconómicas de mayor importancia en comunidades alto andinas (Masson et al., 2016). Tanto es así, que la población de vacunos en el Perú para el 2001 fue de 4512 832 , la de vacas lecheras a nivel nacional fue de 563 757, y la producción de leche promedio a nivel nacional fue de 4,16 kg (Olivera, 2001). En la Región Amazonas, el distrito de Leymebamba cuenta con 614 ganaderos (Oliva et al., 2015). En este sentido, la población en este distrito se dedica mayoritariamente a la ganadería, sin embargo en esta actividad también encontramos algunas limitantes que ponen en riesgo la productividad y la rentabilidad ganadera como es el caso de la salud animal (Vargas et al., 2012), un factor de vital importancia en el sector, ya que de ella también depende la salud de los consumidores (Tafur, 2009).

Al trabajar con un animal que tenga un valor genético superior, el equilibrio en cuanto a la salud parece ser frágil (Ardila, 2010; Martínez et al., 2016). El efecto del clima en el ganado bovino también afecta en su salud, ya sea directa o indirectamente ya que modifica la calidad y/o cantidad de alimentos disponibles, los requerimientos de agua y energía, la cantidad de energía consumida y el uso de esta (Arias, 2008). Dentro de las principales enfermedades en los terneros, encontramos el parasitismo, pierna negra, enfermedades respiratorias y diarreas. Mientras tanto, en el ganado adulto encontramos enfermedades como mastitis, timpanismo o empanzamiento, fiebre de leche y ántrax, entre otros (FAO, 2010).

El uso de plantas en la medicina tradicional del lugar en estudio es una práctica antigua, ya que además de ser usadas para la salud de las personas, también son utilizadas para la salud de los animales (Masika y Afolayan, 2003). No obstante, hoy en día con el desarrollo de la ciencia y la tecnología son pocas las personas que siguen utilizando estas plantas como remedio natural (Betancourt y Nahuelhual, 2017), quedando en un plano secundario dichas tradiciones (Cañedo, 2001). Es por ello que se pretende recuperar estos conocimientos tradicionales para mantener la salud ganadera mediante los conocimientos del productor de bovinos a pequeña escala, basados en la experiencia cotidiana, adquirida en los quehaceres de la actividad pecuaria y en la tradición cultural (Jurado et al., 2007), y de esta manera mejorar la sostenibilidad de los sistemas de pequeños rumiantes para satisfacer la demanda de sostenibilidad (Nardone et al., 2004).

\section{MATERIAL Y MÉTODOS}

\section{Ubicación del área de estudio}

La investigación se desarrolló en el distrito de Leymebamba, perteneciente a la provincia de Chachapoyas, Región Amazonas. Las tres localidades muestreadas dentro de este distrito (Leymebamba, Palmira y Pomacochas) se encuentran ubicadas a una altitud que ronda los 2158 m.s.n.m., en la latitud $06^{\circ} 42^{\prime} 28^{\prime \prime}$ Sur y la longitud $77^{\circ} 48^{\prime} 13$ " Oeste, con una superficie de 373,14 $\mathrm{Km}^{2}$ (Figura 1).

Este proyecto de investigación se desarrolló en las siguientes fases:

\section{Fase preliminar}

En esta fase se hizo una revisión de información referida al proyecto de investigación. También se calculó el número de pobladores ganaderos que existen en el distrito de Leymebamba para extraer la muestra con la que se trabajará en la siguiente fase.

Fase de campo

Para el desarrollo de esta fase se tuvo en cuenta el protocolo para la obtención y clasificación de información etnobotánica y variables socioeconómicas, criterio desarrollado por Cook (1995), con las modificaciones adicionales propuestas por Macía et al. (2011). De esta manera, primero se seleccionaron los anexos de estudio, luego se prepararon los materiales de trabajo antes de las salidas de campo, y posteriormente se aplicaron las entrevistas semiestructuradas.

\section{Fase de gabinete}

Para la elaboración de los mapas de ubicación se hizo uso del software ArcGis v. 10.2. y Google Earth, mientras que para el análisis de datos se utilizaron los programas de Microsoft Excel y SPSS 20.0.7. 


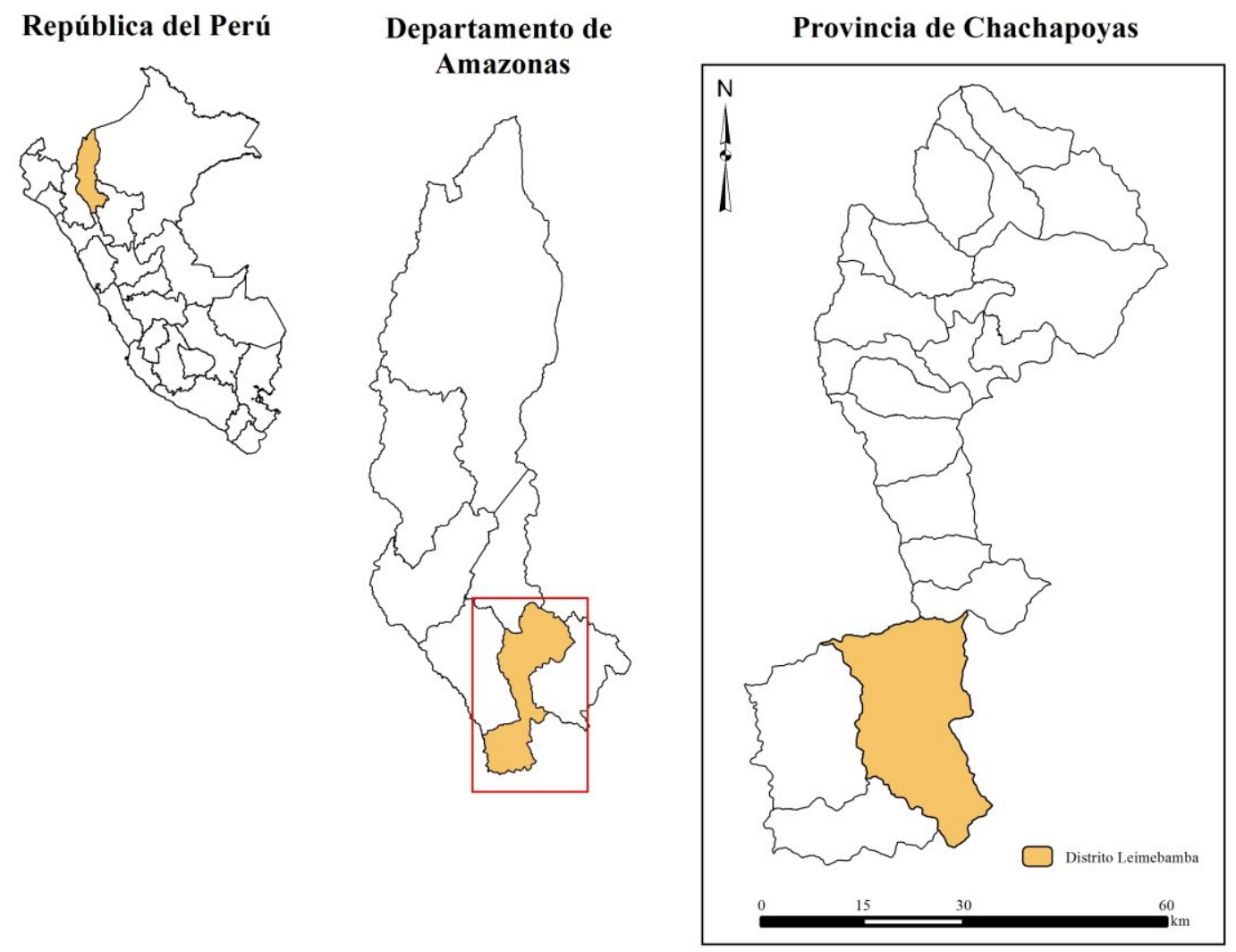

Figura 1. Ubicación de la investigación en el distrito de Leymebamba, provincia de Chachapoyas (Departamento de Amazonas).
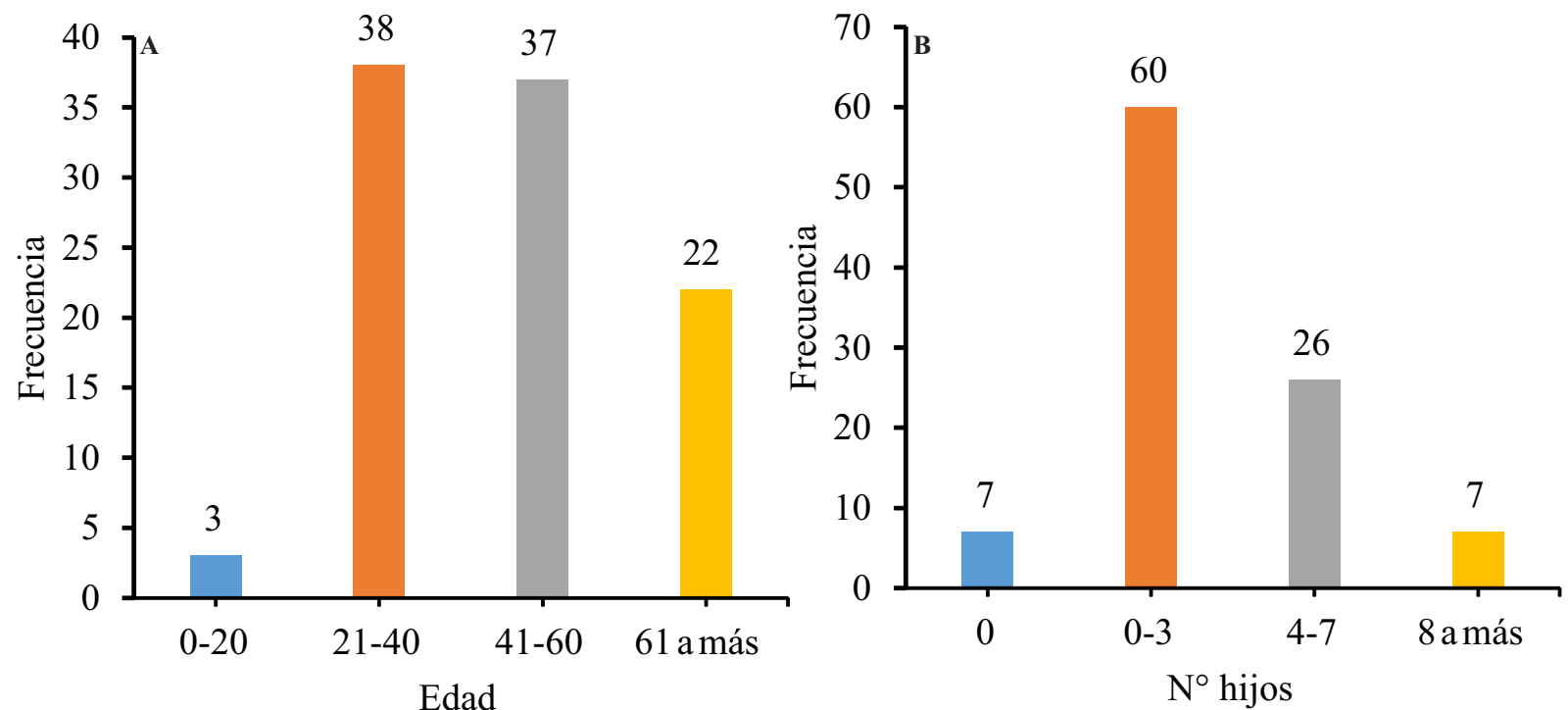

Figura 2. A: Rango de edad de los encuestados en el distrito de Leymebamba; B: N. ${ }^{\circ}$ de hijos de los encuestados.

\section{RESULTADOS}

Caracterización de la población ganadera del distrito de Leymebamba

La población ganadera del distrito de Leymebamba que fue encuestada, se encuentra en el rango de edad entre los 21 a 40 años (Figura2A). El rango de número de hijos de la población ganadera del distrito de
Leymebamba que fue encuestada fue de 0 a 3 hijos (Figura 2B). Mientras que el material predominante de las viviendas en el distrito de Leymebamba es el adobe (Figura 3). 


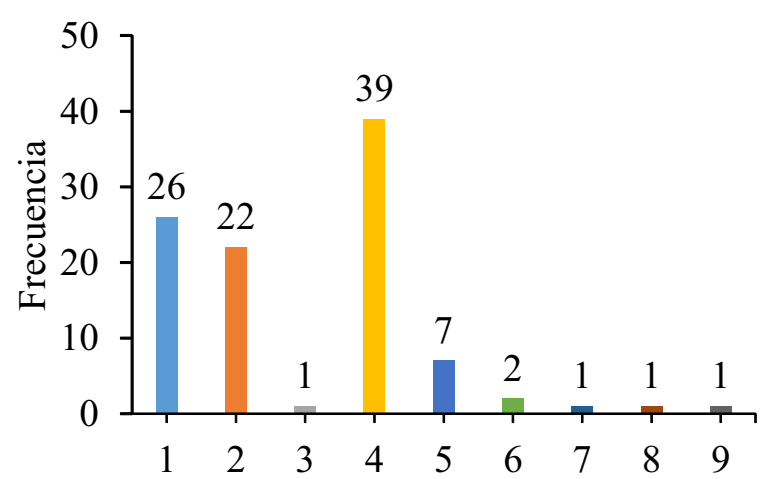

Figura 3. Material de vivienda de los encuestados. (1: Adobe; 2 Cemento; 3: Madera; 4: Otros; 5: Adobe y cemento; 6 : Adobe y otros; 7: Adobe, cemento y otros; 8: Adobe y madera; 9: Madera y otros).

Determinar el conocimiento etnobotánico para el tratamiento de enfermedades de ganado, a nivel de edady género

Después de realizar la tabla de contingencia para los variables género y uso de las plantas medicinales para tratar enfermedades de ganado (Figura 4), se observó que el género femenino es el que mayor uso ejerce sobre las plantas medicinales (25\%).

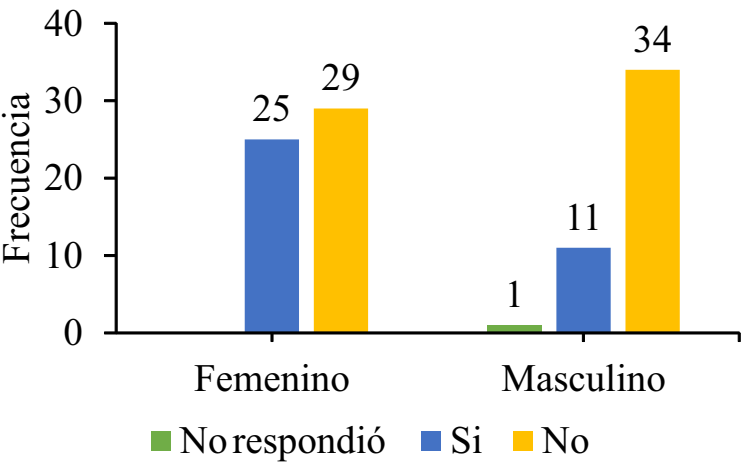

Figura 4. Uso de las plantas medicinales vs género.

Por otra parte, y después de realizar la tabla de contingencia para las variables edad y uso de las plantas medicinales para tratar enfermedades de ganado (Figura 5), se observó que los ganaderos que están en el rango de edad de 21 - 40 años (16\%) son los que más utilizan plantas medicinales. Destaca de igual manera el alto porcentaje de los que no usan plantas medicinales para tratar enfermedades de ganado en todos los rangos de edad. Asimismo, tras realizar la tabla de contingencia para las variables de edad y transmisión de conocimientos, se observó que en el distrito de Leymebamba no existe una tendencia o línea clara de transmisión del mismo a rangos de edad inferiores (Figura 6). También

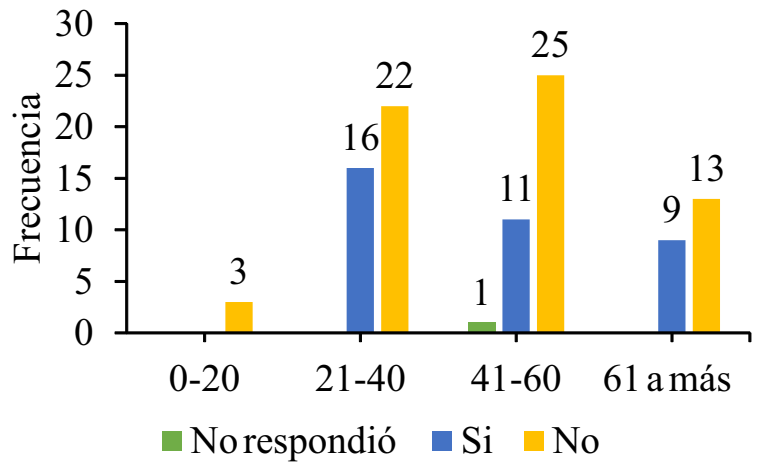

Figura 5. Uso de las plantas medicinales vs edad.

se analizaron las variables género y transmisión de conocimientos con tablas de contingencia, y se observó que en el distrito de Leymebamba no hay transmisión de conocimientos a nivel de género (Figura 7).

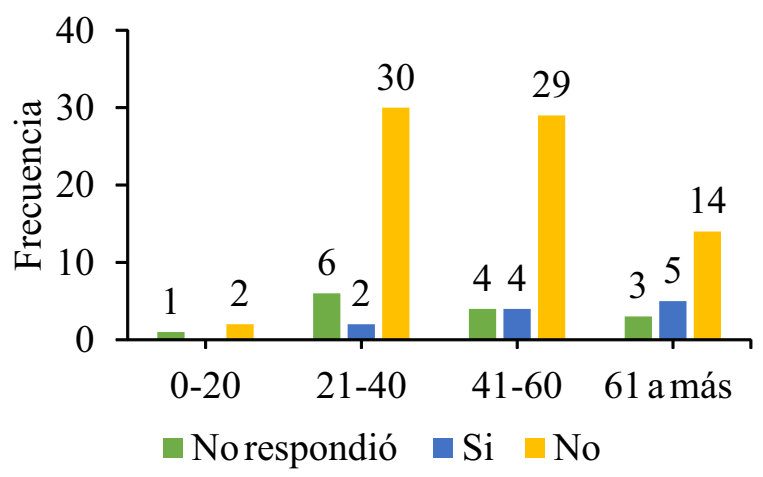

Figura 6. Transmisión de conocimientos vs edad.

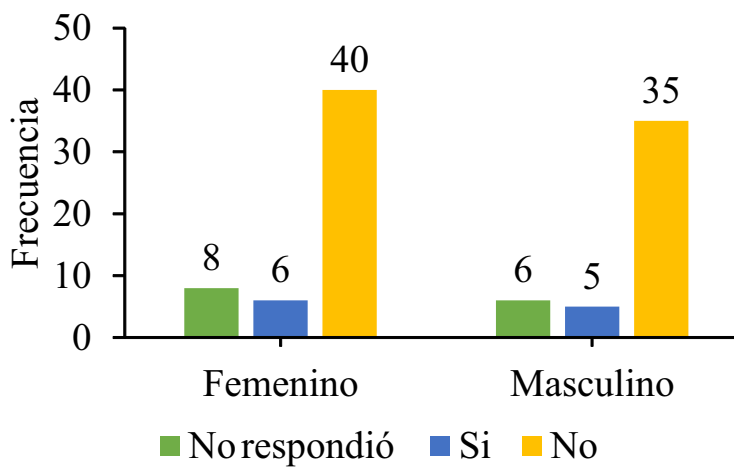

Figura 7. Transmisión de conocimientos vs genero.

Identificación de las principales plantas utilizadas para el tratamiento de enfermedades de ganado

A continuación se indican las principales plantas medicinales usadas en el distrito de Leymebamba para tratamiento de enfermedades y dolencias del ganado, especificando nombre vulgar, nombre científico, indicaciones médicas, parte usada, preparación, modo de administración, estado de la planta, modo y lugar de colecta, y uso presente o pasado (Tabla 1). 


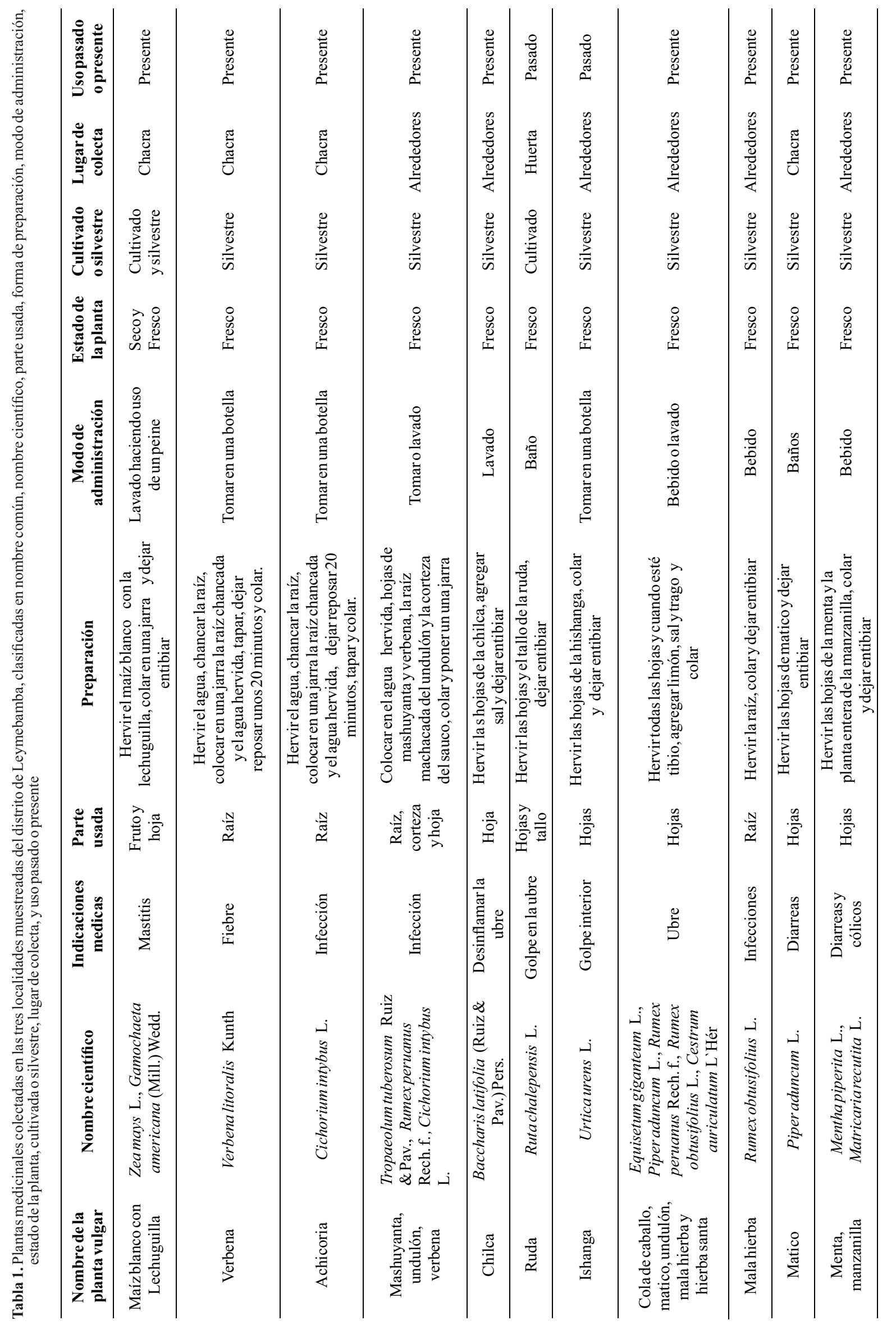




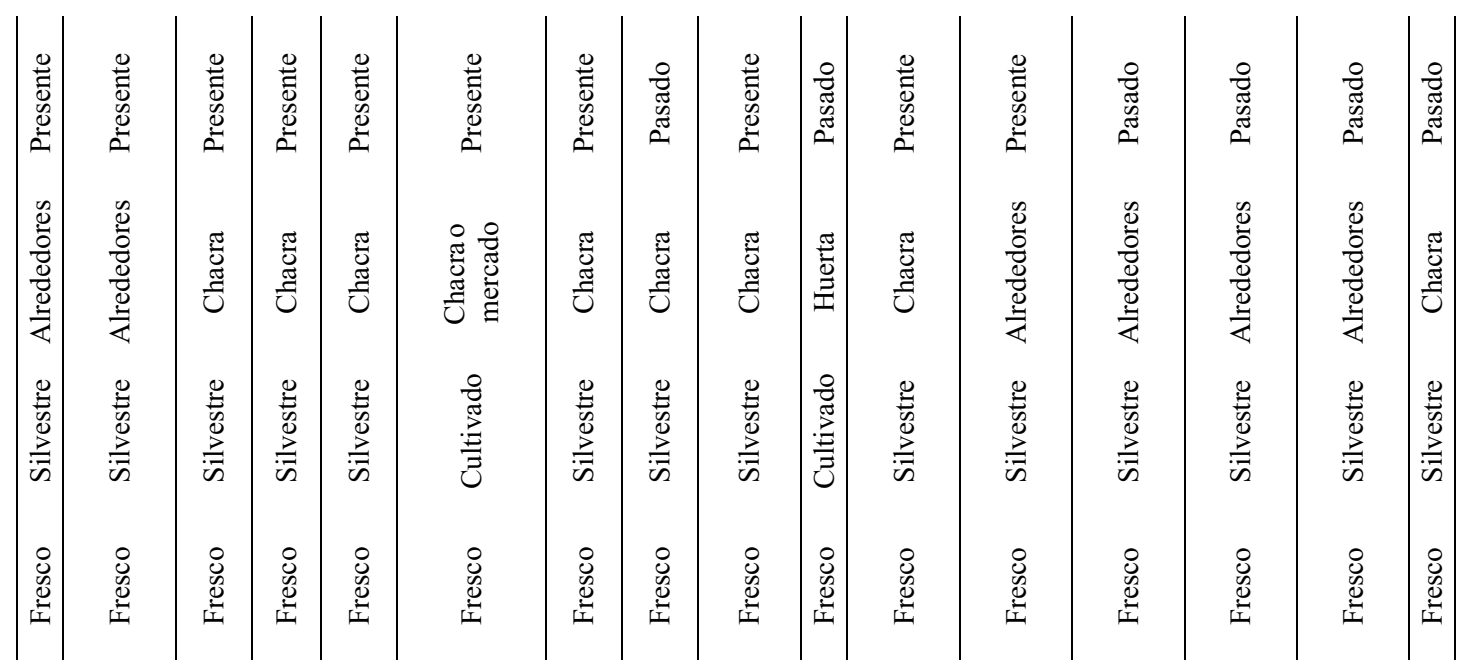

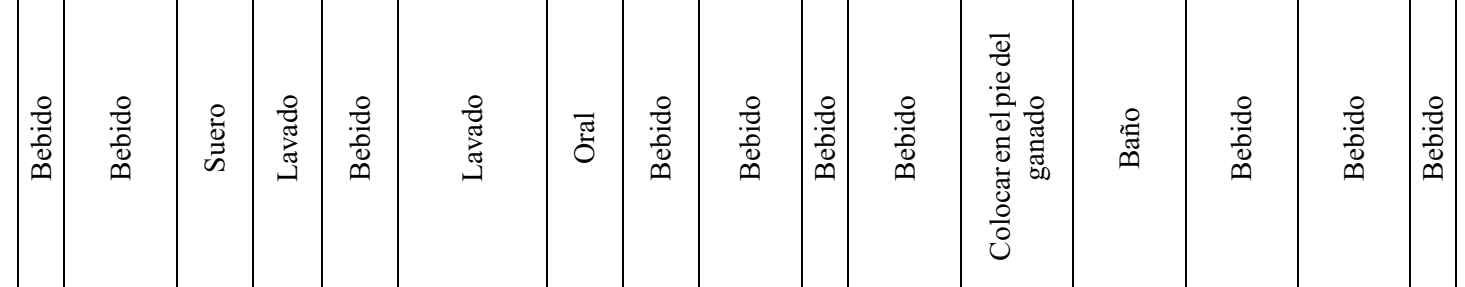

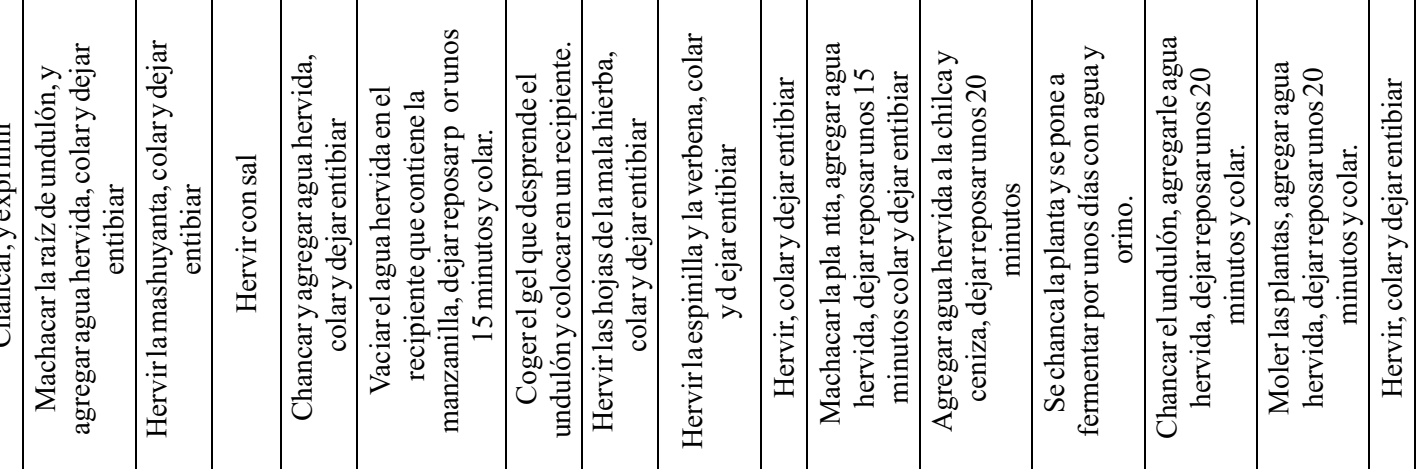

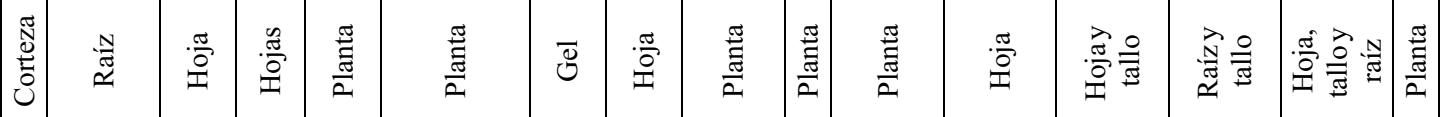

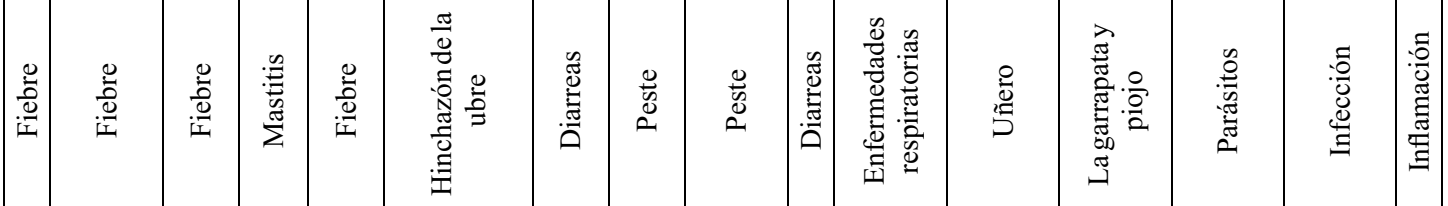

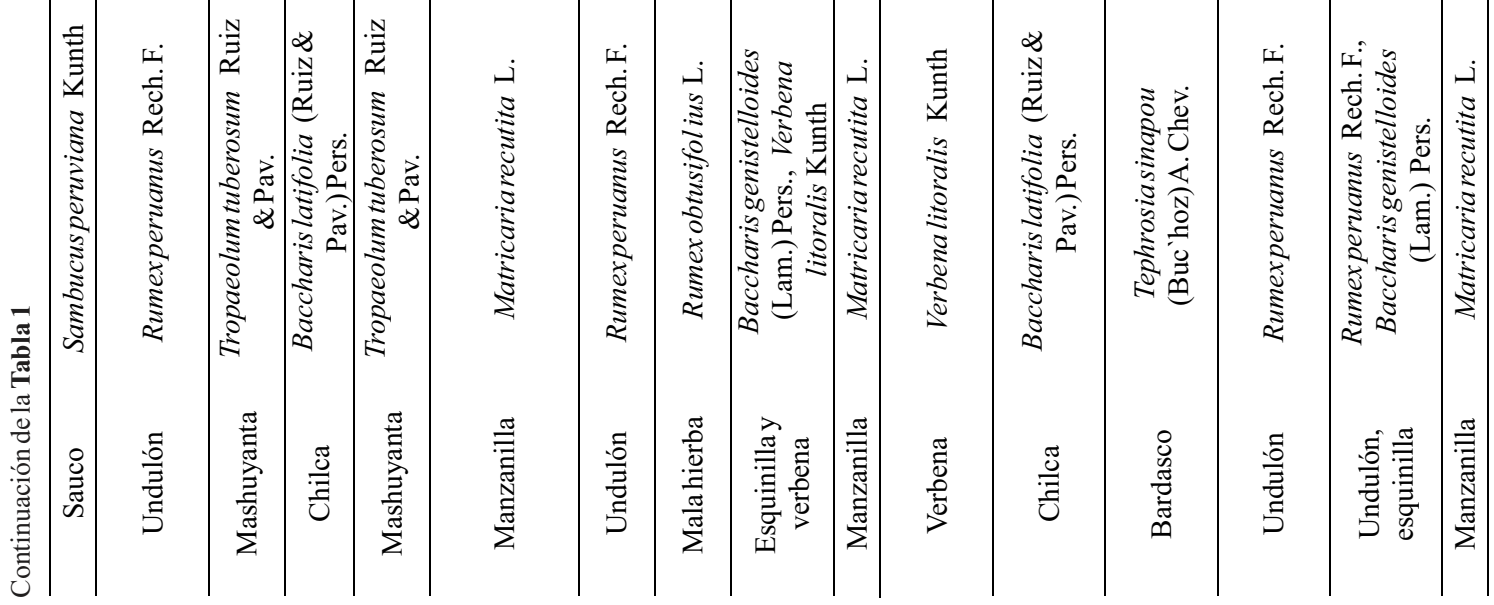




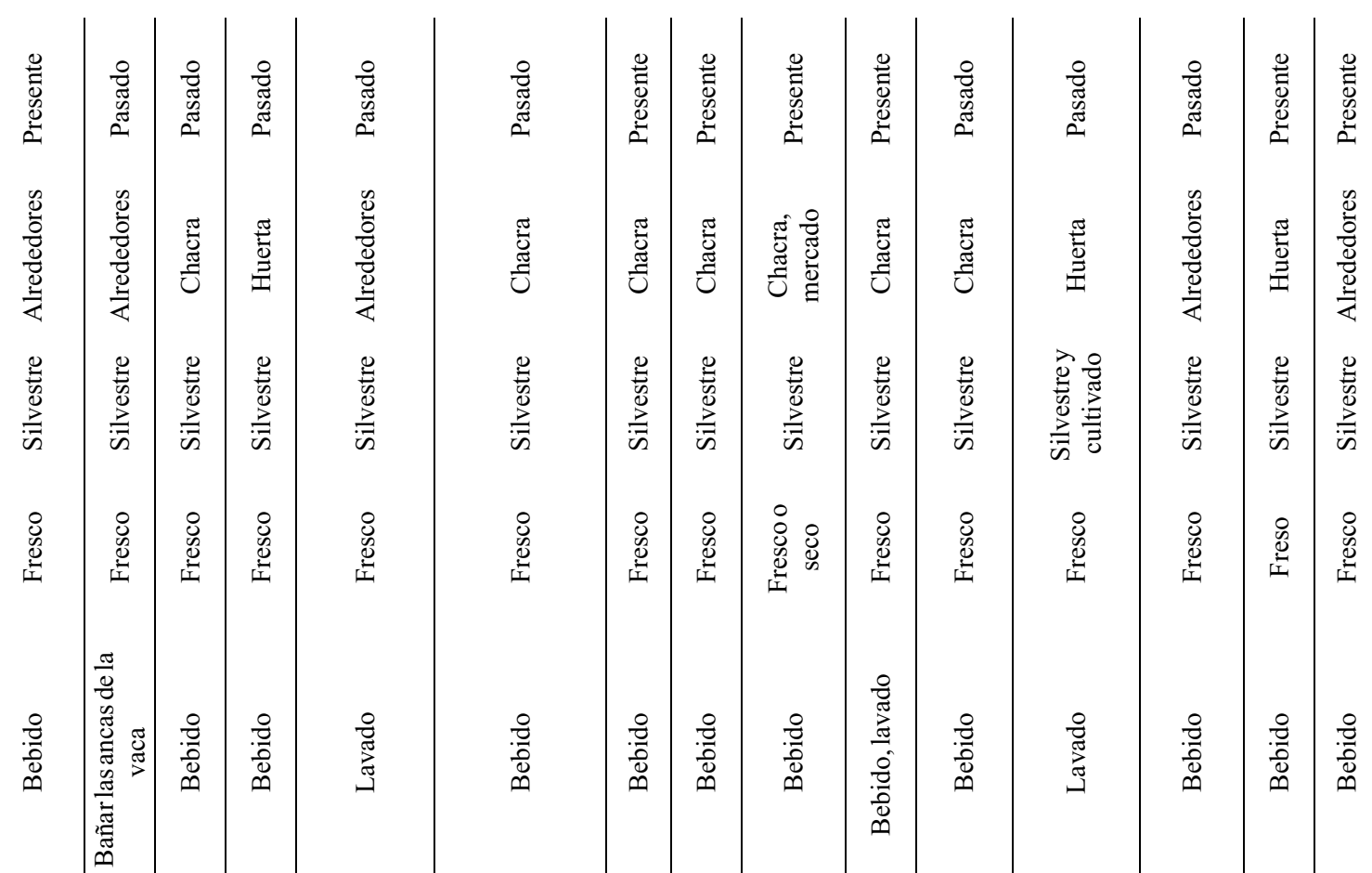

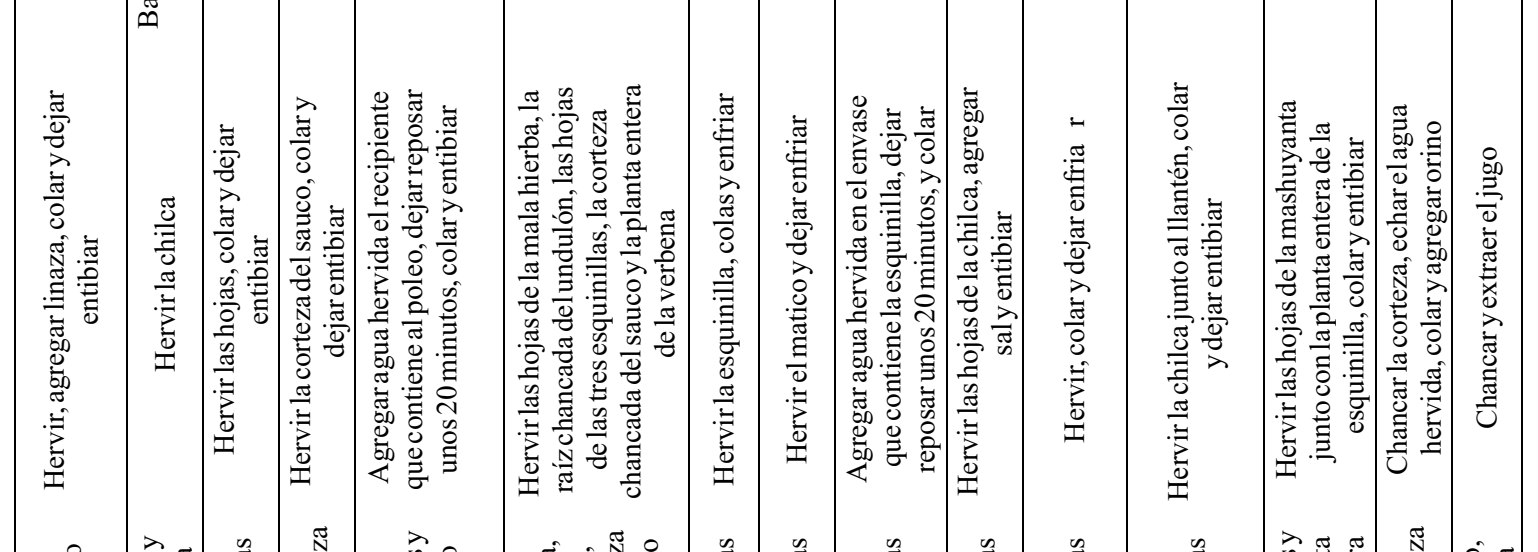

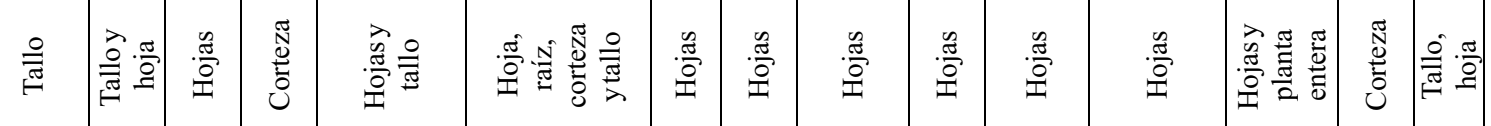

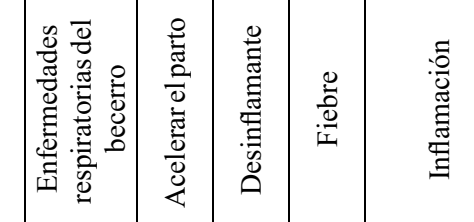

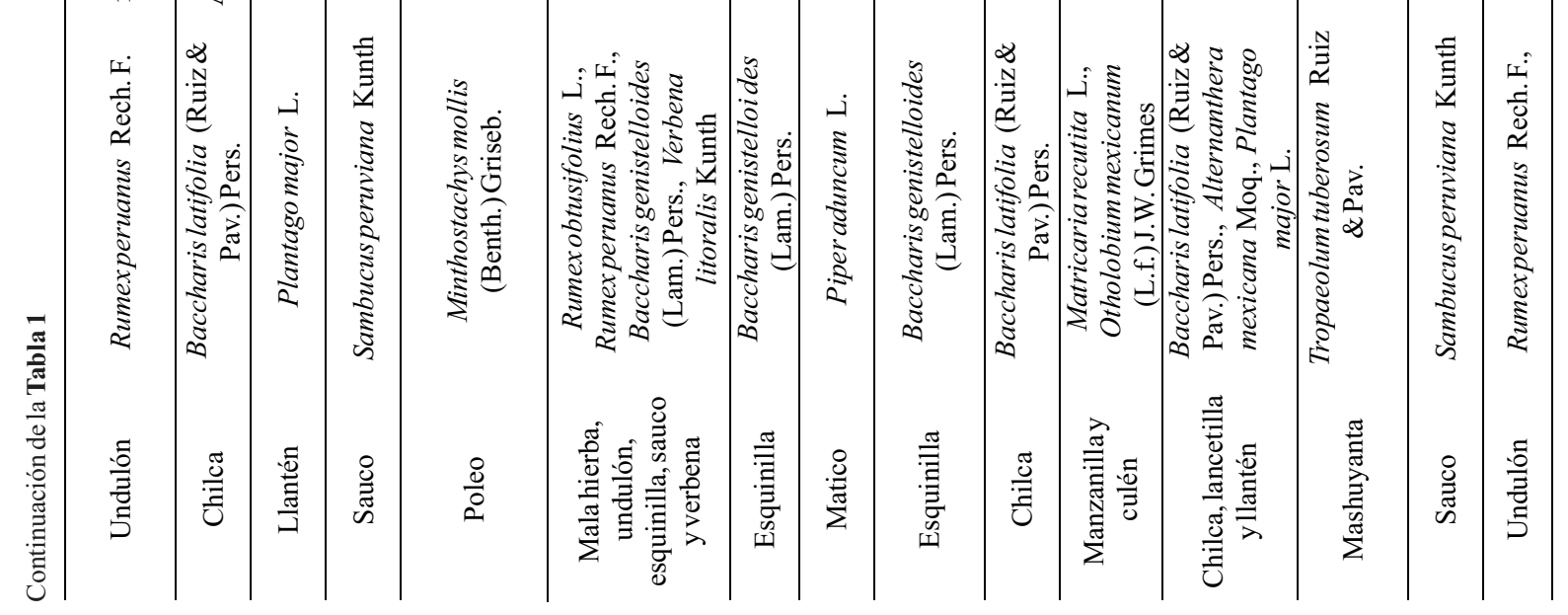




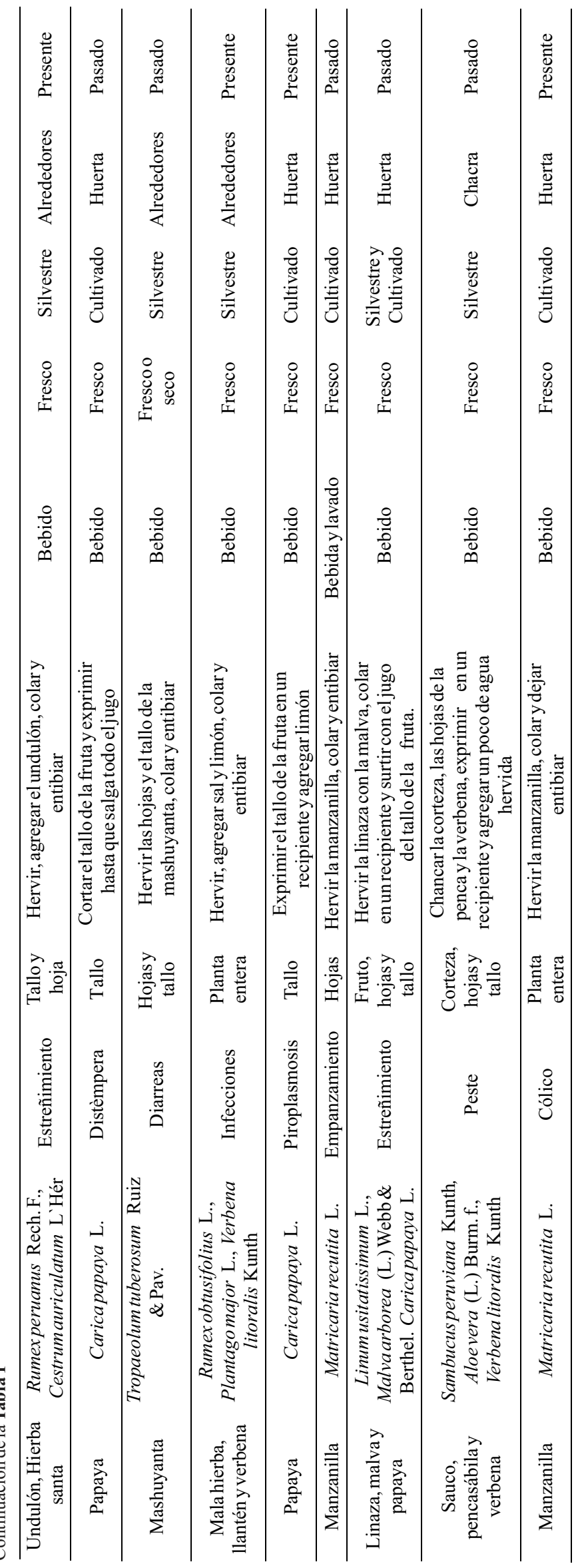


Un total de 26 especies fueron reportadas, colectadas e identificadas en las tres localidades muestreadas. Entre ellas, la más citada fue el undulón (Rumex peruanus Rech. f.), con nueve citaciones. A continuación se situó la verbena (Verbena litoralis Kunth), con 7 reportes, seguida de la mashuyanta (Tropaeolum tuberosum Ruiz \& Pav.) y la esquinilla (Baccharis genistelloides (Lam.) Pers.), ambas con cinco citaciones (Tabla 1).

En cuanto a las partes usadas las hojas fueron las más usadas, con el 58\% de los reportes. La forma de preparación más frecuente fue hervida con el $87 \%$ de las citaciones. El modo de administración más común fue oral o bebido, con el 77\%. El estado de la planta a la hora de su uso fue en su mayoría en fresco (98\%), y su modo de colecta fue principalmente en estado silvestre $(85 \%)$. El lugar de colecta habitual fue la chacra (40\%), seguido de cerca por alrededores del centro poblado (38\%). Finalmente, el uso de estos recursos fue más común en el presente $(58 \%)$ que en el pasado $(42 \%)$.

\section{IV.DISCUSIÓN}

La ganadería es una de las principales actividades para la economía de subsistencia de la población rural del país (Díaz-Ramírez y Oviedo-Angüis, 2013). Sin embargo, existen agentes etiológicos que causan problemas sanitarios al ganado y que pueden repercutir en el consumo salud de los humanos (Castañeda, 2015).

La etnobotánica es una ciencia que nos explica cómo el ser humano hace uso de los recursos naturales, y en este caso vegetales (Carreño, 2016). El uso de plantas medicinales presenta una serie de bondades como el tratamiento de enfermedades de animales, y que son usados por muchas comunidades (Grosso, 2010).

En el distrito de Leymebamba, gran parte de la población hizo uso de plantas medicinales para tratar enfermedades de su ganado. En la actualidad no son muchos los que lo siguen utilizando debido a la introducción de productos de uso agropecuario, consecuencia de la tecnología que prima el interés económico sobre las tradiciones culturales de una comunidad (Jurado et al.,
2007). No obstante, además de la tecnología, son los cambios sociales y económicos los que traen consigo la pérdida de comunicación de los jóvenes con las personas mayores, rompiéndose de este modo la cadena de transmisión oral intergeneracional (Pardo-deSantayana y Gómez, 2003).

En la presente investigación se encontraron muchas plantas, que crecen de manera silvestre y que presentan diversas bondades para tratar las enfermedades del ganado. Sin embargo no se encontraron estudios específicos en relación a las características fitoquímicas o etnobotánicas de las plantas medicinales de esta zona, al igual que en otros lugares, como menciona el estudio elaborado por Moya y Escudero (2015) en Chile.

El número de especies identificadas, 26, es muy inferior a otros realizados y focalizados en áreas de estudio más grandes, como las 351 especies citadas por Bonet y Vallès (2006), en la Región de Cataluña (España), o las 114 especies reportadas en el distrito de Kilte Awulaelo (Etiopía) (Teklay et al., 2013). Sin embargo, este valor si se aproxima a otros estudios etnomedicinales dirigidos al ganado en distintas partes del mundo, como en la provincia de Eastern Cape (Sudáfrica) (Masika y Afolayan, 2003), 41 especies en Rajasthan (Galav et al., 2010), o las 33 especies citadas en la Administración de Dire Dawa (Etiopía) (Kebede et al., 2017).

\section{CONCLUSIONES}

No existe una clara transmisión de conocimientos en la población ganadera del distrito de Leymebamba sobre el uso de plantas medicinales en el ganado, Sin embargo, en términos de género, las mujeres son las que más uso hacen de las plantas medicinales para tratar enfermedades de su ganado.

Dentro de las plantas medicinales más utilizadas para tratar enfermedades de ganado en el distrito de Leymebamba, encontramos la undulón, verbena, esquinilla y mashuyanta, por este orden. Estas plantas son utilizadas en la actualidad mayoritariamente por algunos de los pobladores del distrito, las cuales encuentran principalmente en sus chacras y alrededores de los centros poblados, y que crecen casi en su totalidad de forma silvestre. 


\section{REFERENCIAS BIBLIOGRAFÍA}

Ardilla, A. 2010. "Programa de mejoramiento genético para características económicas en razas cebuinas lecheras". Revista de Medicina Veterinaria (19): 11-20.

Arias, R., T. Mader, y P. Escobar. 2008. "Factores climáticos que afectan el desempeño productivo del ganado bovino de carne y leche". Arch Med Vet 40 (1): 7-22.

Betancourt, R. S. y L. Nahuelhual. 2017. "Servicios ecosistémicos y bienestar local: caso de estudio sobre productos de medicina natural en Panguipulli, sur de Chile". Ecología austral 27 (1): 99-112.

Bonet, M. À. y J. Valles. 2006. "Ethnobotany of Montseny biosphere reserve (Catalonia, Iberian Peninsula): plants used in veterinary medicine". Journal of Ethnopharmacology 110 (1): 130-147.

Cañedo, R. 2001. “Contribuciones cortas Ciencia y Tecnología en la Sociedad. Perspectiva histórico-conceptual". ACIMED9(1): 72-6.

Carreño P. C. 2016. La etnobotánica y su importancia como herramienta para la articulación entre conocimientos ancestrales y cientificos. Tesis de Licenciatura. Universidad distrital Francisco José de Caldas. Bogotá (Colombia).

Castañeda, S. I. 2015. Diagnóstico de agentes etiológicos causantes de problemas sanitarios en ganado lechero en la Parroquia Llagos, Cantón Chunchi, Provincia de Chimborazo. Tesis de Maestría. Escuela Superior Politécnica de Chimborazo. Riobamba (Ecuador).

Cook, F. E. M. 1995. Economic Botany Data Collection Standard. Richmond (Reino Unido): Royal Botanic Gardens Kew.

Díaz-Ramírez, R., y F. Oviedo-Angüis. 2013. Cadena productiva de Ovinos. Lima (Perú): MIGRAMI.

FAO (Food and Agriculture Organization). 2010. Manejo Sanitario Eficiente del Ganado Bovino: Principales enfermedades. Managua
(Nicaragüa): INTA/INATEC.

Galav, P., A. Jain, S. S. Katewa y A. Nag. 2010. “Animal healthcare practices by livestock owners at Pushkar animal fair, Rajasthan." Indian Journal of Traditional Knowledge 9 (3): 581584.

Grosso, L. 2010. El uso popular de las plantas medicinales en Uruguay: La experiencia de los pequeños productores agroecológicos. Milán (Italia): ZooBioDi - Associazione Italiana di Zootecnia Biologica e Biodinamica.

Jurado, C., J. C. Duarte, y O. F. López. 2007. "Recuperación de los conocimientos tradicionales relacionados con la salud de bovinos a pequeña escala en Villamaría, Caldas, Colombia." Vet. Zootec 1 (2): 20-29.

Kebede, A., S. Ayalew, A. Mesfin y G. Mulualem. 2017. "An Ethnoveterinary Study of Medicinal Plants Used for the Management of Livestock Ailments in Selected Kebeles of Dire Dawa Administration, Eastern Ethiopia”. Journal of Plant Sciences. 5 (1): 34-42.

Macía, M. J., P. J. Armesilla, R. Cámara-Leret, N. Paniagua-Zambrana, S. Villalba, H. Balslev, y M. Pardo-de-Santayana. 2011. "Palm uses in northwestern South America: a quantitative review". The Botanical Review 77 (4): 462570.

Martínez, G. M., V. H. Suárez, y M. D. Ghezzi. 2016. "Bienestar animal en bovinos de leche: selección de indicadores vinculados a la salud y producción". Revista de investigaciones agropecuarias 42 (2): 153-160.

Masika, P. J., y A. J. Afolayan. 2003. "An ethnobotanical study of plants used for the treatment of livestock diseases in the Eastern Cape Province, South Africa". Pharmaceutical Biology 41 (1): 16-21.

Masson, M., G. Gutiérrez, V. Puicón, y D. Zárate. 2016. "Helmintiasis y Eimeriosis Gastrointestinal en Alpacas Criadas al Pastoreo en Dos Granjas Comunales de la Región Pasco, Perú, 
y su Relación con el Peso y Condición Corporal”. Rev Inv Vet Perú 27 (4): 805-812.

Moya M.A. y V. G. Escudero. 2015. "Las plantas medicinales en el control de nemátodos gastrointestinales en cabras: potencial de las plantas que crecen en la región de Coquimbo, Chile". Rev. Bras. Pl. Med. 17 (3): 480-494.

Nardone, A., G. Zervas, y B. Ronchi. 2004. "Sustainability of small ruminant organic systems of production". Livestock Production Science 90 (1): 27-39.

Oliva, M., C. Oliva, D. Rojas, M. Oliva y A. Morales. 2015. "Identificación botánica de especies nativas de pastos más importantes de las cuencas lecheras de Molinopampa, Pomacochas y Leymebamba, Amazonas, Perú.” Scientia Agropecuaria 6(2): 125-129.

Olivera, L. 2001. "Sanidad del ganado lechero de la cuenca del sur". Rev Inv Vet Perú, 12 (2) (2001): 78-86.

Pardo-de-Santayana, M., y E. Gómez. 2003. "Etnobotánica: Aprovechamiento tradicional de plantas y patrimonio cultural". Anales Jard. Bot. Madrid 60 (1): 171-182.

Tafur, A. 2009. "La inocuidad de alimentos y el comercio internacional." Rev Colomb Cienc Pecu 22 (3): 330-338.

Teklay, A., B. Abera, y M. Giday. 2013. “An ethnobotanical study of medicinal plants used in Kilte Awulaelo District, Tigray Region of Ethiopia”. Journal of ethnobiology and ethnomedicine 9 (1): 65.

Vargas, D., A. Góngora, y J. J. Correa. 2012. "Enfermedades virales emergentes en ganado de leche de América Latina." Orinoquia 16 (2): 88-96. 